Entrevista 



\title{
"La etapa de evolución social en la que nos encontramos ya está fusionada con la inteligencia artificial, mucho más allá de cualquier punto de retorno"
}

\author{
Entrevista al Dr. Martin Hilbert \\ Manuel Santillán Vásquez \\ Universidad de Lima, Perú \\ masantil@ulima.edu.pe
}

doi: 10.26439/contratexto2018.n029.1962

\begin{abstract}
Resumen. Martin Hilbert es, sin lugar a dudas, uno de los más destacados investigadores de los últimos tiempos. Desde sus trabajos multidisciplinarios publicados en revistas científicas de gran impacto y trayectoria, nos ha hecho posible entender a la sociedad de la información de manera más íntegra. Hilbert ha trabajado para distintos organismos internacionales y se interesa particularmente en el rol de la información o big data en sistemas sociales complejos. Discípulo de Manuel Castells, actualmente, es profesor asociado en el Departamento de Comunicaciones de la Universidad de California. Con esta entrevista, hemos querido ahondar en la forma en que Hilbert ve el impacto de la digitalización en ámbitos como la economía, la investigación, la política, las ciencias sociales y la humanidad, en general.

Palabras clave: transformación digital / inteligencia artificial / big data / deep learning / deep mind / Google
\end{abstract}




\title{
"The current era of social evolution is already merged with artificial intelligence beyond the point of no return"
}

\begin{abstract}
An interview with Dr. Martin Hilbert
Авstract. Without a doubt, Martin Hilbert is one of the most prominent researchers in recent times. Due to his multidisciplinary works published on important scientific journals, it is now possible to understand information society better. Hilbert has worked for many international organizations and he is particularly interested on the role of Big Data and information in complex social systems. He is a disciple of Manuel Castells and, as of now, he is an associate professor for University of California's communications department. This interview aims to delve deeper into Hilbert perceptions on the impact of digitalization on economics, research, politics, social sciences and humankind. Keywords: digital transformation / artificial intelligence / big data / deep learning / deep mind / Google
\end{abstract}




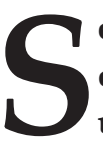

eñor Hilbert, en una entrevista que le hicieron a inicios del 2017, usted mencionaba que hay algo más de 5 zetabytes de información en el mundo y que esta se duplica cada dos años, superando incluso al código genético humano. Con respecto a esa masa de información, ¿en qué categorías se la puede clasificar? ¿Acaso por temas? ¿O qué sistema existe para ordenar esa información? ¿O es que allí es donde ya todo queda en manos de la inteligencia artificial?

Para comenzar, podríamos contextualizar partiendo desde el desarrollo humano mismo, uno que vino en tres grandes etapas: la Edad de Piedra, la Edad de Bronce, la Edad de Hierro. Es entonces cuando creamos o descubrimos conocimientos que nos permitieron crear tecnologías. Esto consistió, al inicio, en básicamente transformar materiales a simples herramientas, pero estas eran las tecnologías que nos ayudaron a progresar. Después, vino una época en que aprendimos a transformar energía, primero de agua, después energía en forma de vapor, lo que llaman la Segunda Revolución Industrial, la electricidad y la máquina de combustión, el automóvil, y todo eso. Y ahora, en las últimas dos ondas largas de evolución humana, llamadas ondas de Kondrátiev, y de acuerdo con la teoría de la innovación schumpeteriana, nos encontramos en una fase en que hemos empezado a transformar información. Es decir, primero material, después energía y ahora transformamos información. Y, dentro de esta última fase, también podemos distinguir dos épocas diferentes. En la primera $-y$ esto lo digo yo-, hemos creado mucha información y comunicación, hemos creado muchos datos con internet, con los celulares, con las bases de datos. Y la segunda es aquella que está más enfocada en el conocimiento, es decir, en una donde extraemos conocimiento de esa información.

Dicho esto, entonces, la respuesta sería sí, hemos creado mucha información hasta ahora y, en esta segunda época, la única manera de aprovechar esto es luchar fuego contra fuego, es decir, con nuestros propios procesadores biológicos, nuestros cerebros, aunque estos no estén preparados para procesar esa cantidad de información, una que hemos producido en los últimos veinte, treinta, cuarenta años. Por eso, la única manera con la que podemos extraer conocimientos, patrones, en este mar de información es con la tecnología misma. A esto se le llama inteligencia artificial, que es mucho mejor que nosotros, que nuestros cerebros.

Por ejemplo, hace un par de años, la gente hubiera dicho: "Ah, ya, pero las máquinas no pueden reconocer caras; nosotros tenemos miles de años de evolución en que hemos aprendido a reconocer caras; entonces, somos expertos y las máquinas no pueden hacer eso". Hoy en día, las máquinas, en los últimos cinco años, son mucho mejores que nosotros en reconocer caras. Las máquinas son mejores que nosotros incluso en reconocer células de cáncer. Ahora puedes mostrar una mancha de 
nacimiento y la inteligencia artificial te puede decir, mejor que cualquier experto humano, si es cancerígena o no. Por tanto, las máquinas son muy buenas porque las entrenamos con mucha más información $\mathrm{y}$ aprenden patrones que nosotros nunca hemos descubierto, ya que nunca nuestro cerebro ha estado expuesto a tanta información para procesarla así como lo hace la inteligencia artificial.

Bueno, y ahora estamos en el momento en que vemos que la inteligencia artificial aprende todo eso, y también de otras inteligencias artificiales, porque simplemente se puede copiar este conocimiento como copiar y pegar; entonces, estas aprenden muy rápidamente y es así como se extrae conocimiento de toda esa información. Esa es la época en la que estamos ahora.

En medio de este proceso de digitalización, ¿cómo se traduce eso en un progreso también para sociedades que no necesariamente pertenecen al mundo desarrollado? ¿Dónde se dan las principales oportunidades para países en vías de desarrollo, cuáles serían los ejes fundamentales sobre los que una sociedad debería actuar o diseñar políticas para avanzar junto a las tecnologías?

Yo creo que la inteligencia artificial trae muchas oportunidades para todos. En previas revoluciones industriales, uno necesitaba materiales o también un conocimiento específico para, por ejemplo, la industria automotriz o para crear plantas nucleares. Pero hoy lo que tenemos es básicamente la posibilidad de crear conocimiento de manera automatizada y desde algo que se encuentra disponible; el aprovechamiento de esto debería provenir de una inteligencia artificial creada localmente.

Por ejemplo, Google y Amazon publican sus códigos de inteligencia artificial. Bueno, lo hacen porque también están bastante asustados, juegan con fuego, y dicen que la mejor manera es "que todos ustedes sepan lo que hacemos". Elon Musk, uno de los más grandes de Silicon Valley, tiene una iniciativa que se llama Open AI, inteligencia artificial abierta, donde se publican estos códigos. Es cierto que no publican cómo entrenaron a esos códigos, pero todos sabemos que ¡los entrenaron con tus datos! Y eso fue lo que Google hizo, entrenó un cerebro artificial por 20 años, Amazon también, $\mathrm{y}$ eso los hace poderosos. Entonces, lo que hacen es como darte un cerebro vacío, pero no te comparten cómo lo entrenaron. Pero al cerebro vacío, igual de potente, puedes acceder. Se puede ir en línea a TensorFlow, que es de Google, y ahí puedes usar la inteligencia artificial que Google ofrece.

A partir de ahí, el máximo valor que se puede sacar de eso es entrenarlo y usarlo con tus propios datos locales. Por ejemplo, cada pyme en América Latina debería entrenar su propio cerebro artificial; una pyme que trabaja en la agricultura o vendiendo zapatos puede entrenar este cerebro con sus propios datos y la inteligencia artificial le hace recomendaciones sobre cómo mejorar sus procesos. Ahora todos estos insumos están disponibles, y son gratis; lo mismo pasa con los datos que cada 
pyme produce con cada acción que hace. Son datos que no solamente provienen del comercio electrónico, sino también del inventario, de la producción, y todo aquello que se encuentre digitalizado. Toda esa información se puede entrenar y llevar a la inteligencia artificial. Con ello, y en comparación con las revoluciones industriales anteriores, la barrera de entrada para aprovecharse de eso es mucho menor porque tenemos la tecnología disponible y el conocimiento.

Para esto se necesita de personal calificado, un recurso humano con el que los países en vías de desarrollo no cuentan.

Ese sería el único cuello de botella: los recursos humanos. Aunque esto siempre fue así, solo que en revoluciones industriales anteriores la diferencia era también la del capital, la falta de conocimiento y las habilidades, pero hoy en día nos faltan solo las habilidades. Estas son bastante fáciles de obtener, ninguno de nosotros aprendió sobre inteligencia artificial en el colegio, porque eso hace quince años no existía, y si existía, no estaba demostrada su utilidad. Los resultados, por ejemplo, en la importancia de deep learning, se han demostrado recién en los últimos cinco años. Es claro que todos nosotros deberíamos aprender eso también y saber de qué se trata. ¿Pero cómo lo hacemos? Pues con cursos en línea, como la mayoría de la gente que vive en Silicon Valley.

Ahora se tiene una barrera de entrada mucho menor y se puede aprender mucho más rápido. Claro, se necesita una capacitación básica de matemática y tal vez de programación, pero eso también se puede aprender, son cosas bastante mecánicas. Entonces, imagínate si todas las pymes en América Latina necesitaran de alguien que entrene un cerebro artificial, requeriríamos a mucha gente. Por eso, yo creo que hay grandes posibilidades de explotar esto en países en vías de desarrollo, pero es cierto que mucho depende de los recursos humanos para explotarlo y sacarle provecho.

Por otro lado, y en el ámbito comercial, sobre todo con relación al crecimiento de las aplicaciones y las redes sociales, sabemos que la información que allí se obtiene es puesta a disposición de terceros. Esto ocurre incluso con nuestro consentimiento cuando aceptamos las políticas y condiciones de uso. Quiero decir que no es un secreto que la data que generamos se termine vendiendo a otros. En ese sentido, iel derecho a la privacidad es una batalla que ya hemos perdido? ¿Hay razón para preocuparse por eso, o mientras más información disponible exista, mejor?

La definición de privacidad está cambiando radicalmente. Hoy en día, las nuevas generaciones ya entienden algo completamente diferente sobre ese aspecto. Tal vez hace veinte años tu orientación sexual era heterosexual, pero no hablabas de eso. Luego vino la definición LGBT, después se agregó intersexuales, queer, asexuales, etcétera. Y ahora, por ejemplo, en el Facebook de Inglaterra hay más de setenta categorías 
con las que tú puedes clasificar tu sexualidad, y los jóvenes lo hacen, los jóvenes se clasifican así; entonces, ellos tienen un entendimiento de privacidad muy distinto del que nosotros teníamos hace veinte años.

Yo creo que, para la educación, para la salud e, incluso, para los negocios, es muy útil conocernos mejor. Aquí, en San Francisco, hay una farmacia donde no hay ninguna persona a cargo, solo robots, y estos son mucho mejores que cualquier estudiante de farmacéutica. Los farmacéuticos estudian muchos años y aun así todavía cometen errores. Los robots nunca han cometido un error, porque conocen el historial médico de la gente, saben exactamente cuándo se pueden dar contraindicaciones. Entonces, saber más sobre tu salud es útil para nosotros, porque hay tantas variables en la salud que, conociéndolas, te pueden salvar la vida.

Ahora, claro que hay que crear instituciones que regulen quién tiene acceso a qué tipo de información sobre ti. Por decir, el robot de la farmacia tiene que saber sobre tu historial clínico, pero tal vez el servicio militar no debería saber sobre eso; ahí hay que hacer una separación. En ese sentido, la pregunta podría ser cómo hacemos para desarrollar instituciones que aseguren quién tiene acceso a qué tipo de información y para qué, o qué tipo de inteligencia artificial será la que tenga acceso a qué. Nosotros ni siquiera necesitamos tener acceso a eso, la información puede estar encriptada; diferentes inteligencias artificiales podrían tener diferentes tipos de accesos a diferentes informaciones. Pero el problema está en que debemos crear instituciones para que esto pueda ser realmente útil para nosotros, en la salud, los negocios, la educación; ahí debemos procurar encontrar las instituciones, hay que trabajar en eso y estoy seguro de que vamos a lograrlo.

Lo que sí me preocupa es la política, porque la democracia no está diseñada para este entorno de información que tenemos. La economía puede evolucionar, la salud va a evolucionar, la educación y la cultura también van a evolucionar, la democracia no, hay que reinventarla completamente. La democracia representativa no cuadra con la realidad informacional en la que vivimos.

Eso queda claro después de saber todo lo que sucedió en las últimas contiendas electorales norteamericanas; algoritmos que pueden predecir la personalidad de las personas por sus "me gusta", o el hecho de crear cientos de miles de perfiles hacia quienes se dirigen mensajes microsegmentados, tantos como sea posible, lo que abre incluso camino a la manipulación. ¿No está usted trazando un panorama desalentador para la evolución de la web y de la democracia?

La verdad es que sobre la democracia podríamos empezar preguntándonos ¿cómo se hace eso hoy? La idea de tener un gobierno de la gente, por la gente, para la gente, tiene un problema de información. Por ejemplo, ¿cómo 
obtengo la información de la gente? ¿Por y para la gente? Eso es algo que pasa por filtros de información.

A lo largo de los milenios hemos ensayado diferentes filtros; este es el caso de la democracia directa, como la tenían en Grecia. Mucho después nos hemos dado cuenta de que con la democracia directa el pueblo es bastante irracional. Max Weber, el padre de la sociología, justamente dijo que "el pueblo es tonto"; el pueblo no puede pensar hasta el día después de mañana; si le das el poder al pueblo, el pueblo te gastaría el presupuesto nacional hasta la hora del almuerzo, después empezarían una guerra con el pueblo de al lado a las tres de la tarde. Bueno, esto es porque nosotros, el pueblo, estamos bastante ocupados todo el día, hacemos muchas cosas, no tenemos la calma de pensar las cosas hasta el final; entonces, para eso inventamos la democracia representativa. Y estos representantes representan no mi opinión sobre algo, sino mi razonamiento, y ahí les damos un mandato libre. En principio, yo elijo a alguien en quien confío, en alguien que llegaría a la misma conclusión si yo estuviera dedicando mucho tiempo a deliberar sobre un tema en especial. Y así, ese filtro de información que tiene la democracia representativa, en forma de Congreso, Senado, funcionó muy bien hasta que la tecnología digital los destruyó.

Hoy en día, no es un mandato libre; hoy en día demandamos que el político haga exactamente lo que nosotros le decimos, y el político, al mismo tiempo, verá en Twitter cuál es la opinión del pueblo y hará sus políticas de acuerdo con la opinión del pueblo, bajo transparencia completa. Mientras el político da una charla, tiene feedback en tiempo real, revisa las reacciones en Twitter, ajusta su charla, sabe exactamente cómo mandar sus mensajes, dice cosas diferentes a personas diferentes. $\mathrm{Y}$, es cierto, esto no es nada nuevo; en la era digital se manda un mensaje político en doscientas mil versiones diferentes, según el perfil psicológico de los miedos de la gente. La misma frase en doscientas mil diferentes versiones, según los miedos psicológicos de la gente. Se dice lo que la gente quiere escuchar. Hoy, eso no es democracia, eso tiene otro nombre, se llama populismo, y esa es además la razón por la que hay muchos populistas que llegan al poder, por ejemplo, en Estados Unidos.

Nuestra democracia representativa no funciona porque nosotros tratamos de controlar a nuestros representantes, y ellos, a su vez, tratan de controlar y lavar nuestros cerebros. Entonces, así no funciona. Tampoco la democracia directa es la panacea, porque ya hemos visto que no funciona. De ahí, entonces, habría que preguntarnos: ¿cómo hay que reinventar la democracia? No sé, no tengo la respuesta. Pero yo sé que los filtros de información que hemos inventado en el pasado, directa o representativa, ya no funcionan.

¿Pero, por lo menos, hay algún camino, un horizonte esperanzador por el cual 
-al igual que en la salud, la educación y los negocios- se pueda avanzar?

La buena noticia podría ser que, cuando hablamos de información, de la inteligencia artificial y las redes neuronales robóticas, lo que sucede es un aprendizaje informacional muy complejo, multidimensional, y donde se pueden detectar patrones que nosotros con nuestros pequeños cerebros no podemos advertir. Y esos patrones nos dicen: "Mira, acá hay una opinión útil, y se puede hacer eso", ya no hay necesidad de ir a votar. Podríamos preguntar a la gente: "Reforma de educación, escríbame sobre lo que le importa", y después tomamos toda esa información. Para Perú, por ejemplo, tendríamos 30 millones de respuestas, y se las damos a la inteligencia artificial y le decimos que busque un patrón sobre lo que le importa a la gente. Y ahí empezamos a diseñar y a hacer políticas.

Podríamos usar toda esa información cualitativa, una que hace 250 años se sometió a un filtro de información para tener gobernanza de la gente, por la gente y para la gente; por eso se redujo todo el flujo de información a un voto. Hoy tenemos muchas más oportunidades, incluso podríamos pedirle a la gente una opinión cualitativa sobre algún tema y encontrar en qué patrones coincide.

Entonces, si la democracia es un problema de información, tendríamos que pensar un poco más allá y, de repente, plantear cosas más provocadoras como reemplazar el Congreso por una red neuronal artificial, si consideramos que sus tareas son muy similares a lo que hacen las redes neuronales. Filtran información de diferentes niveles hasta que encuentran consenso o desacuerdo. ¿Podríamos hacer eso, reemplazar el Congreso? No sé, pero lo que digo es que deberíamos apuntar a tener una gobernanza de la gente, por la gente, para la gente, y que eso de momento no cuadra con la realidad en la que vivimos.

Se puede notar claramente en la bibliografía que el estudio de las transformaciones digitales y los impactos socioculturales van en dos líneas: aquellas que entienden los fenómenos generados por la web como un constructo tecnológico y aquellos estudios con un enfoque proveniente de las humanidades y las ciencias sociales. ¿Dónde se ubican sus investigaciones? ¿Qué línea siguen?

Lo que creo que está ocurriendo es una fusión de ambas. Por ejemplo, aquí en la universidad, humanidades es una disciplina cualitativa, no cuantitativa, en el sentido de que no trabajan con números ni modelos matemáticos; se realizan estudios empíricos, donde analizan datos. Y, recién ahora, contrataron a tres profesores de big data, estadísticos, especialistas en computación. Lo que han hecho las humanidades, en gran parte, es leer libros. Ahora todos los libros están en Google Books, y puedes, si quieres, preguntar a una inteligencia artificial, que puede leer toda esa data, si es posible encontrar patrones que nosotros nunca descubriríamos. Entonces, como ves, las humanidades también 
tienen unas grandes oportunidades de entender las cosas mucho mejor, a través de la lectura de libros digitalizados.

Entonces, sí, las humanidades también aprovecharán esto bastante, y para mí se están fusionando mucho. Debemos ver que la big data convierte las verdades de las ciencias sociales en una ciencia. Y es que antes, en las ciencias sociales, se obtenían resultados con una varianza del $20 \%$, y con ello lograban publicar en las revistas académicas de sociología más importantes. Pero hacer políticas públicas basadas en un conocimiento proveniente de un $20 \%$ es bastante peligroso. Por eso es que los físicos, los biólogos, siempre nos han dicho que nosotros no somos ciencia, porque apenas entendemos un $20 \%$. Pero ahora, con la big data, sí podemos hacer predicciones del 80 o $90 \%$. Por ejemplo, si tengo tu huella digital y sé dónde estás con tu teléfono, yo puedo predecir con un $90 \%$ de probabilidad dónde vas a estar en dos años.

En el mundo, normalmente se han realizado censos cada diez años; un censo cada diez años es muy caro. Ahora, con tu información en el celular - todo el mundo en América Latina tiene un celular-, es posible tener un censo en tiempo real con un $80 \%$ de precisión. Como ves, podemos hacer mucha más ciencia social que antes; saber, por ejemplo, cuánta gente somos y hacer otras predicciones.

Entonces, la ciencia social realmente se convierte en ciencia. Por eso se dice que lo que fue el telescopio para la astronomía o lo que fue el microscopio para la biología, es el big data para las ciencias sociales. La ciencia social está fusionando la información cualitativa con la cuantitativa, ambos vamos en la misma dirección y eso trae muchas oportunidades.

¿Por qué se dice que la inteligencia artificial y el deep mind estarán definiendo el futuro de cómo se gobernará o cómo se diseñarán los negocios? De ser esto así, todo parecería indicar que muchas tareas pasarán a automatizarse, ¿cuáles serán esas, o qué profesiones se verán afectadas inicialmente? ¿Qué cosas deberíamos dejar a la inteligencia artificial y qué cosas no?

Una de las cosas más interesantes de esta revolución es que no afecta a los trabajadores de cuello azul o al trabajo manual en sí, en contraste con revoluciones industriales anteriores, donde justamente se automatizó el trabajo de estos trabajadores. La revolución de la inteligencia artificial no distingue tan bien ese reemplazo, incluso sustituye parte de los trabajadores de cuello blanco, de trabajos técnicos, de dirección y administración.

Así, les hablé de los profesionales farmacéuticos que, a pesar de tener un entrenamiento y especialización por varios años, están siendo reemplazados por la inteligencia artificial que les supera en eficiencia. Eso mismo está ocurriendo con profesionales como abogados o con expertos en reconocer células de cáncer, incluso haciéndolo mucho mejor. Pero 
esto es algo que siempre ha pasado y va a seguir pasando. Y sobre su pregunta: ¿qué pasará después?, la verdad es que no sé lo que pasará después, siempre en la evolución de la civilización ha habido reemplazos.

Por ejemplo, pensemos en culturas ancestrales en América Latina. Antes se usaba gran parte del tiempo en cultivar maíz, cacao, frejoles, verduras, papas, y se tenía que buscar agua todo el día para regar. Mucha gente se ocupaba de eso, muchos recursos humanos. Hasta que se desarrollaron los acueductos. Estos básicamente automatizaron este proceso de regar para la agricultura, y esto fue un alivio grande. Entonces, toda esta gente se desocupó porque se automatizó un proceso. Y luego ¿qué empezó a hacer la gente con todo este tiempo libre en sus manos? Algunos se dedicaron a la arquitectura y construyeron estructuras grandes como Machu Picchu. Otros empezaron a estudiar las estrellas. Otros desarrollaron formas de escritura, los famosos quipus, los nudos hablantes, o se hicieron guerreros y empezaron a explorar.

Así, la evolución nos muestra que desarrollamos cosas que antes no nos podíamos imaginar, porque no teníamos el tiempo. Y hoy nosotros estamos muy ocupados con levantarnos en la mañana, comer, irnos al trabajo, sentarnos a trabajar, almorzar con el colega, irnos de vuelta, trabajar, etcétera. Y después, el fin de semana, las dos semanas de vacaciones al año. Bajo esas circunstancias, no podemos imaginarnos las cosas que podríamos inventar, así como le ocurrió a la gente en la Edad de Piedra, ellos no se podían imaginar qué utilidad tendría la escritura. Hoy sabemos que tiene mucha utilidad $y$ hemos avanzado bastante.

Por eso, no te puedo decir qué vamos a hacer, pero yo sé que el universo y la realidad son infinitos, y hay muchas cosas que todavía no hemos explorado, y que podemos explorar. Yo creo que hay mucho después, cosas que ni siquiera sabemos, nos encontramos así como cuando la gente llevaba agua para regar al maíz, sin siquiera saber qué podría pasar una vez que inventemos acueductos. Pensar que nosotros somos la créme de la créme y que hemos llegado hasta el final sería muy arrogante.

Entonces, sí, hay muchas cosas por explorar, y las inteligencias artificiales nos van a ayudar a llegar ahí. Pero sí, el futuro va a ser diferente, no vamos a ir al trabajo ocho horas, no vamos a sentarnos en el auto cuarenta minutos para llegar al trabajo, para trabajar ocho horas, para estar cuarenta minutos en el auto de vuelta, qué pérdida de tiempo, ¿no? No vamos a hacer eso, va a ser diferente. Pero vamos a liberar el tiempo. Con un auto que nos maneje. Con una realidad virtual donde ni siquiera tengamos que irnos cuarenta minutos hacia el trabajo, nos ahorraremos dos horas al día. ¿Qué vamos a hacer? ¿Qué vamos a inventar con eso? No sé. Pero sí, va a ser algo interesante. 
Tomando lo dicho, ¿no deberíamos ser nosotros los humanos quienes definamos el futuro o, por lo menos, tracemos el camino de las convergencias entre tecnologías y sociedad? Lo que parece es que nadie se ocupa de tocar el tema, de iniciar el diálogo o de ponerse a pensar sobre lo que viene después o las implicancias del desarrollo tecnológico. ¿Qué consideraciones centrales deberíamos plantearnos sobre este punto? ¿Sobre qué hay que empezar a hablar?

Sí, las preocupaciones nos llevan rápidamente a un futuro con terminators y a la Matrix que va a venir y va a destruirnos. Sí, yo creo que sí hay que preocuparse sobre eso. En la evolución, ya hemos venido fusionándonos con la tecnología. El 80 \% de nuestras decisiones de distribución de recursos financieros son tomadas por inteligencia artificial. En la bolsa, el $80 \%$ de las decisiones están en poder de inteligencia artificial. El 99,9\% de las decisiones de distribución de energía en la red eléctrica son tomadas por inteligencia artificial, es porque la energía no se puede guardar y tiene que ser distribuida muy rápido, y el cerebro no puede hacer eso. Es la inteligencia artificial quien decide quién tiene energía, cuánto y cómo. Y más de la mitad de matrimonios aquí en este país se deciden a través de la inteligencia artificial, porque hacen un match en online dating; las citas en línea producen más de la mitad de matrimonios aquí (Estados Unidos).

Ahora, si tú me dices que encontramos una especie y, en esta especie, el
$80 \%$ de las decisiones de recursos, el $99 \%$ de las decisiones de energía y un $50 \%$ de las decisiones de procreación son tomadas por esta cosa que llamamos inteligencia artificial, yo le diría, mira, ya son lo mismo. Esto ya es parte de eso. La singularidad tecnológica ya está aquí. Con todo, tú podrías irte a las montañas, deshacerte de tu celular, nunca más tener interacción directa o indirecta con dinero - porque el dinero está manejado por redes digitales- e, incluso, podrías sobrevivir por unos años, en las montañas, ahí, solo. Pero de ninguna manera podrías proclamar que estás coevolucionando con el resto de la sociedad. Tú vives ahí, de vuelta a la Edad Media en las montañas, tú no estás evolucionando con nosotros. La etapa de evolución social en la que actualmente estamos ya está fusionada con la inteligencia artificial, mucho más allá de cualquier punto de retorno.

Precisamente sobre ese aspecto, una de las ideas que usted sostiene llama especialmente la atención, y es aquella donde indica que la digitalización podría estar convirtiéndonos en células de un organismo mayor o que de alguna manera estamos convergiendo con la tecnología para crear un ente superior. ¿Cómo se puede explicar eso y cómo nos podemos imaginar ese ente u organismo mayor?

Normalmente, cuando se fusionan dos especies, en la gran mayoría de los casos, en la evolución hay selección, la selección natural. Sobrevive solamente el 
más apto. La evolución funciona porque todos los que no son tan aptos, o son un poco menos aptos que los otros, mueren. Ahora, hay también algunos ejemplos de simbiosis, y eso es muy importante.

Por ejemplo, la mitocondria y el cloroplasto fueron dos bacterias separadas que hicieron una simbiosis que ahora conforman lo que nosotros llamamos células. Se unieron estas dos bacterias y crearon algo mayor, células. Ahora, nosotros existimos únicamente de células. Entonces, esta simbiosis de dos organismos diferentes dio paso a algo más grande. Y ahora nosotros esperamos que, cuando la inteligencia biológica y la inteligencia artificial se unan, pase algo similar. Bueno, y esperamos que eso sea algo que se vea bien para nosotros. Lo bueno es que, si somos conscientes, podemos empezar a diseñar esa unión o mezcla, que, como ya lo he dicho, ya está sucediendo en el campo social. Y la pregunta es ¿cómo la diseñamos para aprovecharla de una manera óptima?

En este punto, podríamos considerar que la inteligencia artificial hace algunas cosas mejor que nosotros, y algunas cosas nosotros hacemos muy mal. Mira la Tierra, cómo la tratamos. La verdad, eso es imperdonable, es un asco cómo tratamos a la Tierra, no somos buenos, no somos responsables. Entonces, sí, algo de ayuda podríamos necesitar. Pero ¿cómo diseñar eso?, ¿cómo nos mezclamos con eso? Una simbiosis por diseño es muy importante, y tienes toda la razón, deberíamos hablar más de eso. Ahora, todo ese proceso tiene también algunas limitaciones; el filósofo Nick Bostrom, de Oxford, tiene un libro que se llama Superinteligencia, que les recomiendo leer, es un libro muy bueno, donde habla de eso, de la fusión de la inteligencia biológica con la artificial.

Entonces, sí, es muy difícil prepararnos para lo que venga, porque no sabemos lo que viene; lo mejor que podemos hacer es estar muy conscientes y preparados, sobre todo mantener un diálogo constante sobre eso. Mirarlo bien, aprovecharlo, empoderarnos de eso también. Como decía, si cada pyme en América Latina tiene un experto en inteligencia artificial, ahí ya nos hemos fusionado con esa tecnología. Y ahí tenemos que ir rápido, porque la tecnología se desarrolla tan rápido que no podemos esperar. Y lo mejor que podemos hacer es tener entrevistas como esta, que son también parte de todo aquello.

\section{Muchas gracias por la entrevista, señor Hilbert.}

